\title{
Integrated analysis of transcriptome profiling predicts potential IncRNA and circRNA targets in human nasopharyngeal carcinoma
}

\author{
DONG-NI ZHOU ${ }^{1}$, CHUN-SHENG YE ${ }^{2}$, QING-QING YANG ${ }^{2}$ and YAN-FEI DENG ${ }^{2,3}$ \\ Departments of ${ }^{1}$ Pathology and ${ }^{2}$ Otolaryngology-Head and Neck Surgery, Zhongshan Hospital, Xiamen University, \\ Xiamen, Fujian 361004; ${ }^{3}$ Department of Otolaryngology-Head and Neck Surgery, Union School of Clinical Medicine, \\ Fujian Medical University, Fuzhou, Fujian 350001, P.R. China
}

Received April 10, 2019; Accepted November 8, 2019

DOI: $10.3892 / 01.2020 .11412$

\begin{abstract}
Non-coding RNAs (ncRNAs) regulate numerous genes and influence the progression of various human diseases, including cancer. The role of regulatory ncRNAs implicated in nasopharyngeal carcinoma (NPC), as well as their target genes, remains unclear. The present study aimed to investigate specific long non-coding (lnc)RNAs, circular RNAs (circRNAs) and mRNAs associated with the molecular pathogenesis of NPC, and to predict the underlying target genes of specific lncRNAs and circRNAs. The expression levels of lncRNAs, circRNAs and mRNAs in NPC and chronic nasopharyngitis tissues were detected and analyzed using microarray and bioinformatics techniques. A total of $2.80 \%$ lncRNAs (425 upregulated and 431 downregulated) were significantly differentially expressed (DE) between the two tissue types. Additionally, $0.96 \%$ circRNAs (18 upregulated and 13 downregulated) were significantly DE, while $2.94 \%$ mRNAs (426 upregulated and 341 downregulated) were significantly DE between the two tissue types. In total, 420 NPC-associated nearby encoding genes (196 up- and 224 downregulated) of the DE lncRNAs were identified. Overlap analysis identified 23 DE circRNAs and their corresponding target genes, with 37 microRNAs and 50 mRNAs, from which 14 interaction networks were constructed. Subsequent pathway analysis revealed $221 \mathrm{DE}$ target genes corresponding to 31 key signaling pathways associated with NPC, 14 of which may represent hub genes associated with NPC pathophysiology. Thus, certain lncRNAs, circRNAs and mRNAs are aberrantly expressed in NPC tissues, and partially specific lncRNAs, circRNAs and their target genes may influence the tumorigenesis and progression
\end{abstract}

Correspondence to: Professor Yan-Fei Deng, Department of Otolaryngology-Head and Neck Surgery, Zhongshan Hospital, Xiamen University, 209 Hubin South Road, Xiamen, Fujian 361004, P.R. China

E-mail: dyanfei@139.com

Key words: nasopharyngeal carcinoma, long non-coding RNA, circular RNA, microRNA, mRNA, microarray, bioinformatics analysis of NPC. Target prediction and regulatory network identification may help to determine the pathogenic mechanisms of NPC.

\section{Introduction}

Nasopharyngeal carcinoma (NPC) is the most prevalent malignancy in southern China and Southeast Asia. In total, $\sim 129,000$ new cases are reported annually worldwide, with $>70 \%$ reported in South China and Southeast Asia (1). Its pathogenesis is associated with three primary etiological factors; Epstein-Barr virus infection, genetic susceptibility and environmental condition (2). However, the pathophysiological mechanism underlying NPC progression is yet to be elucidated.

Previous studies have predominantly focused on the function of specific genes expressed in NPC instead of the molecular pathogenesis of the disease $(1,2)$. Notably, $>98 \%$ of human genes are non-protein coding, and the expression of these genes generates non-coding RNAs (ncRNAs) (3). There are multiple families of ncRNA, including ribosomal (r), short interfering (si), micro (mi), circular (circ) and long non-coding (lnc)RNA (4-6). The latter refers to ncRNAs $>200$ nucleotides in length $(4,5)$. Previous studies have reported that lncRNAs are important regulators of numerous biological processes, including tumor progression $(7,8)$. miRNAs are a class of small ncRNAs of $\sim 22$ nucleotides, which serve as key regulators of multiple disease-associated processes (9). Unlike linear RNA, circRNAs exhibit a covalently closed continuous loop, and serve as an miRNA sponge to regulate transcription (10). A number of studies have indicated that ncRNAs play important roles in transcriptional regulation by forming regulatory networks and subsequently interacting with their respective target genes (4-7).

lncRNAs and miRNAs have become increasingly associated with the progression of NPC (11); however, the majority of studies have solely focused on the role of single or small groups of these molecules. Ma et al (12) reported that lncRNA HOX transcript antisense RNA contributes towards the tumorigenesis of NPC via the upregulation of fatty acid synthase. Moreover, Zhang et al (13) discovered that miRNA-200c acts as an oncogene in NPC, by regulating the phosphatase and tensin homolog genes. To the best of our knowledge, a 
limited number of studies to date have investigated the association between circRNA expression and NPC, and no data are currently available concerning circRNAs and their target genes in NPC. Shuai et al (14) indicated that circRNA_0000285 may be used as a novel biomarker for NPC radiosensitivity. As the initiation and progression of different types of tumor is a multi-gene, multi-step process, research is typically focused on gene regulatory networks at a genome-wide level.

The purpose of the present study was to comprehensively analyze the expression profiles of lncRNAs, circRNAs and mRNAs in NPC, and their inter-regulatory molecular mechanisms. It was also aimed to identify the target genes of differentially expressed (DE) lncRNAs and circRNAs, and the DE genes (DEGs) within key signaling pathways influencing NPC progression, in order to elucidate the regulatory network in NPC. First, the present study compared the transcriptome profiling of lncRNAs, circRNAs and mRNAs between NPC and chronic nasopharyngitis (CNP) tissues, using microarray technology at the whole genome level. Subsequently, integrated bioinformatics analysis was performed on the three microarray datasets. The results of the present study may help to clarify the associations between IncRNAs, circRNAs and miRNAs (and their target genes), and elucidate the notable regulatory networks involved in the molecular pathogenesis of NPC.

\section{Materials and methods}

Specimens. A total of 42 human nasopharyngeal tissue samples were collected from 42 patients (30 men and 12 women) during nasopharyngeal biopsy between August 2013 and October 2014, at Zhongshan Hospital (Ximen, China). The tissue samples included 21 cases of primary NPC and 21 cases of CNP from patients suspected of having cancer. All specimens were confirmed by histopathological examination. Patients did not receive chemoradiotherapy and biotherapy prior to biopsy. The 21 patients with primary NPC comprised 15 men and six women (age range, 20-69 years; median, 45.5 years), and the 21 patients with CNP comprised 16 men and five women (age range, 21-60 years; median, 43.2 years).

All tissues were immediately stored at $-80^{\circ} \mathrm{C}$ following biopsy, prior to subsequent RNA extraction. Tumor tissues were isolated via micro-dissection and specimens containing $>70 \%$ tumor cells were further analyzed. In total, six pairs of NPC and CNP specimens among the 42 collected tissues were selected for IncRNA, mRNA and circRNA expression microarray, while all 42 specimens were used for reverse transcription-quantitative (RT-q)PCR. These specimens were used to evaluate differences in the expression levels between NPC and CNP tissues. The present study was approved by the Medical Ethics Committee of Zhongshan Hospital, Xiamen University (Fujian, China) and written informed consent was obtained from all patients prior to the study start.

RNA extraction and quality control. Total RNA was extracted from each tissue sample using TRIzol ${ }^{\circledR}$ reagent (Invitrogen; Thermo Fisher Scientific, Inc.) and purified using the RNeasy Mini kit (Qiagen $\mathrm{GmbH}$ ), according to the manufacturers' protocol. The quantity and quality of the RNA were determined using a NanoDrop ND-1000 spectrophotometer (NanoDrop Technologies; Thermo Fisher Scientific, Inc.) and the RNA integrity was assessed via electrophoresis on a denaturing agarose gel.

RNA labeling, IncRNA and mRNA microarray. Sample labeling and microarray hybridization were performed using a modified version of the Agilent One-Color Microarray-Based Gene Expression Analysis protocol (Agilent Technologies, Inc.). The rRNA was removed from the total RNA sample using the Ribo-Zero $^{\mathrm{TM}}$ rRNA Removal kit (Epicentre; Illumina, Inc.) and mRNA was purified using the mRNA-ONLY ${ }^{\text {тм }}$ Eukaryotic mRNA Isolation kit (Epicentre; Illumina, Inc.). Subsequently, each sample was amplified and transcribed into fluorescent cRNA along the entire length of the transcripts, using a random priming method. The labeled cRNAs were purified using the RNeasy Mini kit (Qiagen $\mathrm{GmbH}$ ), and the concentration and specific activity of the labeled cRNAs [pmol cyanine (Cy)3/ $\mu \mathrm{g}$ cRNA] were measured using NanoDrop ND-1000 (NanoDrop Technologies; Thermo Fisher Scientific, Inc.). A total of $0.6 \mu \mathrm{g}$ of each labeled cRNA was fragmented via the addition of $5 \mu \mathrm{l}$ $10 \mathrm{X}$ blocking agent and $1 \mu \mathrm{l} 25 \mathrm{X}$ fragmentation buffer. The mixture was heated at $60^{\circ} \mathrm{C}$ for $30 \mathrm{~min}$, and $25 \mu 12 \mathrm{X}$ GEx hybridization buffer (Agilent Technologies, Inc.) was added to dilute the labeled cRNA. A total of $40 \mu l$ hybridization solution was dispensed into a gasket slide and placed into the Human LncRNA Array (version 3.0; 8x60 K; Arraystar, Inc.), which contained 30,586 lncRNAs and 26,109 mRNAs. The slides were incubated for $17 \mathrm{~h}$ at $65^{\circ} \mathrm{C}$ in an Agilent Microarray hybridization oven. The hybridized arrays were washed with Gene Expression Wash Buffer (Agilent Technologies, Inc.) and subsequently fixed with $3.7 \%$ paraformaldehyde for $15 \mathrm{~min}$ at room temperature prior to being scanned using the Agilent DNA Microarray Scanner System (G2505C; Agilent Technologies, Inc.).

Agilent Feature Extraction software (version 11.0.1.1; Agilent Technologies, Inc.) was used to analyze the acquired array images. Quantile normalization and subsequent data processing were performed using the GeneSpring GX software package (version 11.5.1; Agilent Technologies, Inc.). Following quantile normalization of the raw data, lncRNAs and mRNAs that were flagged as Present or Marginal ('All Targets Value'), in $\geq 6$ out of 12 samples, were selected for further analyses. Significantly DE lncRNAs and mRNAs (fold-change $\geq 2.0$; $\mathrm{P} \leq 0.05$ ) between the two groups were identified via volcano plot filtering. Hierarchical clustering was performed using the Agilent GeneSpring GX software (version 11.5.1; Agilent Technologies, Inc.). Gene Ontology (GO; http://geneontology. org) and Kyoto Encyclopedia of Genes and Genomes (KEGG; http://www.genome.jp/kegg) pathway analyses were performed using the standard enrichment computation method for DE mRNAs. GO analysis was performed in order to characterize genes and gene products in terms of cellular component, molecular function and biological process. KEGG pathway analysis was performed to identify the signaling pathways in which DE mRNAs underwent significant enrichment, and thus predict the underlying biological functions of the DEGs. $\mathrm{P}<0.05$ and the false discovery rate denoted the significance of the GO term enrichment and the biological pathways. The computational data analysis was performed by Kangchen BioTech Co., Ltd. 
RNA labeling and circRNA microarray. Sample labeling and Arraystar Human circRNA Array hybridization (Arraystar, Inc.) were performed according to the manufacturer's protocol. Briefly, circRNAs were treated with RNase R (Epicentre; Illumina, Inc.) to remove the linear RNA. Each sample was subsequently amplified and transcribed into fluorescent cRNA using the Arraystar Super RNA Labeling kit (Arraystar, Inc.) and a random priming method. The labeled cRNAs were purified using the RNeasy Mini kit (Qiagen $\mathrm{GmbH}$ ) and the concentration and specific activity of the labeled cRNAs (pmol Cy3/ $\mu \mathrm{g}$ cRNA) was determined using NanoDrop ND-1000 (NanoDrop Technologies; Thermo Fisher Scientific, Inc.). A total of $1 \mu \mathrm{g}$ of each labeled cRNA was fragmented using $5 \mu \mathrm{l} 10 \mathrm{X}$ blocking agent and $1 \mu 125 \mathrm{X}$ fragmentation buffer. The sample was heated at $60^{\circ} \mathrm{C}$ for $30 \mathrm{~min}$, and $25 \mu 12 \mathrm{X}$ hybridization buffer (Agilent Technologies, Inc.) was added to dilute the labeled cRNAs. A total of $50 \mu \mathrm{l}$ of hybridization solution was dispensed into a gasket slide and assembled into the Arraystar Human CircRNA Microarray slide (Arraystar, Inc.). The slides were incubated for $17 \mathrm{~h}$ at $65^{\circ} \mathrm{C}$ in an Agilent hybridization oven. The hybridized arrays were washed with Gene Expression Wash Buffer (Agilent Technologies, Inc.) and subsequently fixed in $3.7 \%$ paraformaldehyde for $15 \mathrm{~min}$ at room temperature, prior to being scanned using the Agilent Microarray Scanner System (Agilent Technologies, Inc.).

The scanned images were imported into Agilent Feature Extraction software (version 11.0.1.1; Agilent Technologies, Inc.) for raw data extraction. Quantile normalization of the raw data and subsequent data processing were performed using the R software package (version 3.28.0; http://bioconductor. org/packages/edgeR). Low-intensity filtering was performed and circRNAs that were flagged as Present or Marginal ('All Targets Value') in $\geq 6$ out of 12 samples were retained for further analyses. The fold-change between the groups for each circRNA was computed to allow for comparisons between two groups of profile differences (such as cancer vs. inflammation). circRNAs with a fold-change $\geq 1.5$ and $\mathrm{P} \leq 0.05$ were selected as significantly DE. The analysis outputs were filtered and the DE circRNAs were ranked according to their fold-change and P-value using Microsoft Excel's Data/Sort \& Filter functionalities (Microsoft Corporation). The computational data analysis was performed by Kangchen BioTech Co., Ltd.

$R T$ - $q P C R$ validation. Randomly selected DE lncRNAs, mRNAs and circRNAs were evaluated using RT-qPCR. The specific primer sequences for 12 lncRNAs, eight mRNAs and four circRNAs were designed using Primer (version 5.0; Premier Biosoft, Inc.) and are presented in Table SI. The total RNA $(1.5 \mu \mathrm{g})$ was reverse transcribed into cDNA using the PrimeScript ${ }^{\mathrm{TM}} \mathrm{RT}$ Reagent kit (Takara Bio, Inc.), according to the manufacturer's protocol. qPCR was performed on a total reaction volume of $10 \mu \mathrm{l}$, comprised of $5 \mu 12 \mathrm{X}$ Master Mix (Arraystar, Inc.), $0.5 \mu 1$ each of the PCR forward and reverse primers $(10 \mu \mathrm{M}), 2 \mu 1$ DNA and $2 \mu 1$ double-distilled water. The following thermocycling conditions were used for RT-qPCR: An initial denaturation step of $10 \mathrm{~min}$ at $95^{\circ} \mathrm{C}$, followed by 40 cycles of $95^{\circ} \mathrm{C}$ for $10 \mathrm{sec}$ and $60^{\circ} \mathrm{C}$ for $1 \mathrm{~min}$. All experiments were performed in triplicate. For RT-qPCR validation analysis, all 42 samples were normalized to GAPDH. The fold-change in expression was calculated using the $2^{-\Delta \Delta \mathrm{Cq}}$ method (15).

Identification of the nearby coding genes of DE lncRNAs. A nearby coding gene is defined as a coding transcript $<300 \mathrm{~kb}$ between the DE IncRNA and the neighboring coding mRNA. In the present study, genomic coordinate analysis of DE lncRNAs was performed alongside computational analysis of lncRNA and mRNA microarray data. The NPC-associated DE lncRNAs and their neighboring coding genes were annotated, and genomic coordinates of the IncRNAs, and the association between an IncRNA and its nearby coding gene were also detailed. Additionally, nearby coding genes of DE IncRNAs in NPC were obtained following lncRNA classification, subgroup analysis and genomic coordinate determination.

Predictive analysis of potential targets of DE circRNAs. Overlap analysis was performed via three steps. First, the circRNA-miRNA interaction was predicted with Arraystar's proprietary miRNA target prediction software (version 1.0) using datasets retrieved from the TargetScan and miRanda databases, and the DE circRNAs within all the comparisons were annotated in detail using the circRNA-miRNA interaction information. A total of five target miRNAs for each DE circRNA were subsequently identified according to the number of conservative miRNA binding sites. Subsequently, the candidate target mRNAs for the selected target miRNAs of DE circRNAs were analyzed using Overlap software (version 1.0; Kangchen BioTech Co., Ltd.), based on three miRNA databases (miRanda, miRDB and TargetScan). The intersection of the mRNAs between the aforementioned candidate mRNAs, and the mRNAs in the IncRNA and mRNA microarray data, was determined using Venny software (version 2.1; http://bioinfogp.cnb.csic.es/tools/venny/index.html).

Following the determination of DE circRNAs and their corresponding target genes, the circRNA-miRNA-mRNA regulatory network was constructed and visualized using Cytoscape software (version 3.7.1; http://www.cytoscape.org).

Statistical analysis. The differences in IncRNA, circRNA and mRNA expression levels between NPC and CNP tissues (from the microarray and RT-q-PCR data) were analyzed using the paired Student's t-test, according to their fold-change. Fisher's exact test was used for GO and pathway analyses. $\mathrm{P}<0.05$ was considered to indicate a statistically significant difference. For the microarray analysis, the false discovery rate was calculated to correct the P-value.

\section{Results}

Profiles of DE lncRNAs and mRNAs. Among all the lncRNA and mRNA probes in the microarray, $2.80 \%(856 / 30,586)$ lncRNAs (425 upregulated and 431 downregulated) and $2.94 \%(767 / 26,109)$ mRNAs (426 upregulated and 341 downregulated) were significantly DE between the two groups (Tables SII and SIII). The top 20 most significantly DE lncRNAs consisted of more upregulated lncRNAs compared with downregulated lncRNAs (ratio, 16:4). Furthermore, uc004ebm.1 (fold-change, 38.478134) was the most significantly 

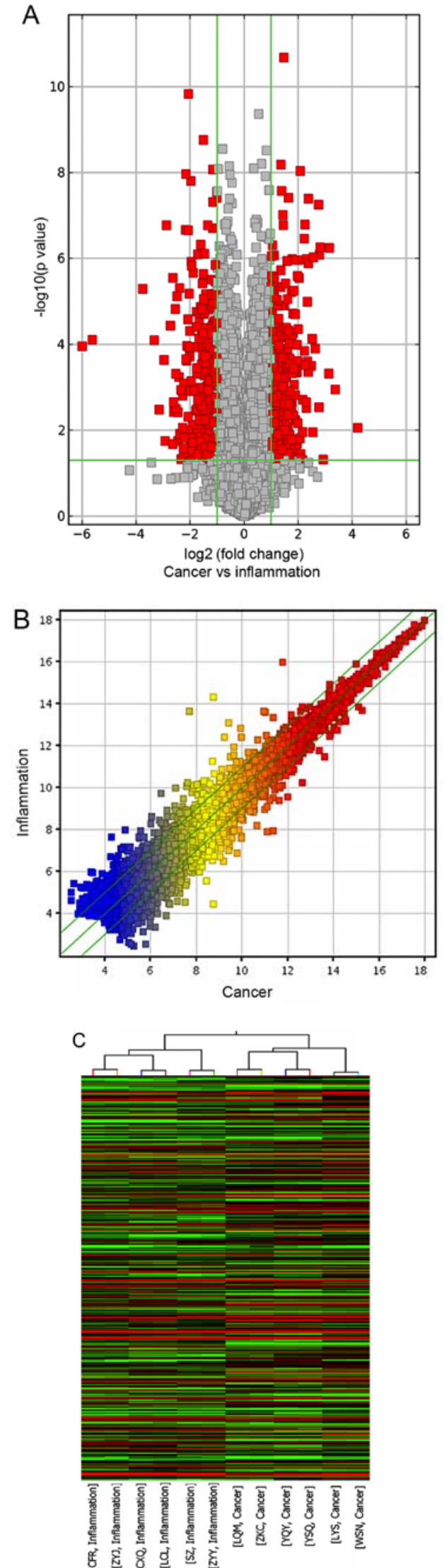

Figure 1. Bioinformatics analysis of differentially expressed mRNAs in NPC and CNP tissues. (A) Volcano plot demonstrating the distribution of mRNAs. (B) Scatter plot exhibiting variation in mRNA expression. (C) Heatmap clustering indicating a distinguishable association between the mRNA expression patterns of certain samples. mRNA expression levels are indicated as follows; Red, high expression and green, low expression. NPC, nasopharyngeal carcinoma; CNP, chronic nasopharyngitis. upregulated lncRNA, while ENST00000572818 was the most significantly downregulated lncRNA (fold-change, 12.04174). Of the 20 most significantly DE mRNAs; the number of upregulated and downregulated mRNAs were equal (ratio, 10:10). Furthermore, SPRR2E (fold-change, 63.81733) was the most significantly downregulated mRNA, while PCDH10 (fold-change, 18.465176) was the most significantly upregulated mRNA. Differences in mRNA expression between NPC and CNP tissues are presented using a volcano plot, scatter plot and clustering heat-map (Fig. 1A-C, respectively). In addition, pathway analysis revealed that 31 significantly enriched signaling pathways (18 upregulated and 13 downregulated) corresponded to DE mRNAs in this microarray (Tables I, II, SIV and SV).

Profiles of DE circRNAs. In the circRNA microarray, $0.96 \%$ of all probes (31 circRNAs, 18 upregulated and 13 downregulated) were significantly DE between the NPC and CNP samples (Tables III and SVI). These comprised two circRNAs (upregulated); hsa_circRNA_100160 (circRNA identifier) and hsa_circRNA_100989, with >6-fold-change differences in expression. The variation of circRNAs between NPC and CNP tissues is presented in a volcano plot, scatter plot and clustering heat map (Fig. 2A-C, respectively).

Validation of selected DE lncRNAs, mRNAs and circRNAs using RT-qPCR. Of the 12 selected DE lncRNAs (NR_027358, ENST00000566575,ENST00000560280,ENST00000411815, ENST00000565929, ENST00000457799, ENST00000514571, ENST00000440518, uc010zye.1, ENST00000443373, ENST00000513638 and ENST00000429469), only ENST00000560280 exhibited significantly opposing microarray expression patterns, which was compared with those resulting from RT-q-PCR analysis. Changes in the expression levels of the other 11 lncRNAs were also verified by RT-q-PCR (Fig. 3A). Of the 8 selected DE mRNAs (SHISA8, XAF1, RORA, MEF2C, TXLNB, MCM2, STAT1 and caspase 3 ), the expression levels of all except MCM2 exhibited the same trends of up- and downregulation as the microarray data, which indicated a significant difference between NPC and CNP tissues, following RT-qPCR (Fig. 3B). The changes in expression of the four selected DE circRNAs (hsa_circRNA_104204, hsa_circRNA_101252, hsa_circRNA_100160 and hsa_circRNA_001430) were consistent with the results of microarray analysis (Fig. 3C).

Prediction of potential targets of DE lncRNAs. Following bioinformatics and genomic coordinate analyses, the NPC-associated nearby coding genes of DE lncRNAs were listed in the relevant tables (Tables IV, V, SII, SVII and SVIII), including their association and corresponding gene accession number, gene name, protein name, gene strand, gene start location and gene end location. In summary, 420 NPC-associated nearby coding genes, corresponding with DE lncRNAs, were accurately defined and comprised 196 upregulated and 224 downregulated genes.

Prediction of potential targets within significant pathway. In total, 31 significantly enriched signaling pathways (18 associated with upregulated and 13 associated with downregulated 
Table I. Key signaling pathways associated with upregulated DEGs.

\begin{tabular}{|c|c|c|}
\hline Pathway ID & Definition & DEGs \\
\hline hsa04060 & $\begin{array}{l}\text { Cytokine-cytokine receptor interaction- } \\
\text { Homo sapiens (human) }\end{array}$ & $\begin{array}{l}\text { CCL2, CCL4, CCR8, CXCL10, CXCL2, CXCL3, CXCL6, } \\
\text { CXCR6, EGFR, FAS, GHR, IFNG, IL12A, IL15, IL22RA2, } \\
\text { IL23A, LIFR, TNFRSF11B, TNFSF10, TNFSF18 }\end{array}$ \\
\hline hsa05164 & Influenza A-Homo sapiens (human) & $\begin{array}{l}\text { CCL2, CXCL10, EIF2AK2, FAS, IFIH1, IFNG, IL12A, } \\
\text { MX1, OAS1, OAS2, RSAD2, STAT1, TMPRSS13, } \\
\text { TNFSF10 }\end{array}$ \\
\hline hsa05162 & Measles-Homo sapiens (human) & $\begin{array}{l}\text { EIF2AK2, FAS, IFIH1, IFNG, IL12A, MX1, OAS1, OAS2, } \\
\text { STAT1, TNFSF10 }\end{array}$ \\
\hline hsa00260 & $\begin{array}{l}\text { Glycine, serine and threonine } \\
\text { metabolism-Homo sapiens (human) }\end{array}$ & CHDH, GATM, PIPOX, PSAT1, SDS \\
\hline hsa04630 & $\begin{array}{l}\text { Jak-STAT signaling pathway-Homo sapiens } \\
\text { (human) }\end{array}$ & $\begin{array}{l}\text { GHR, IFNG, IL12A, IL13RA2, IL15, IL22RA2, IL23A, } \\
\text { LIFR, SPRY2, STAT1 }\end{array}$ \\
\hline hsa05160 & Hepatitis C-Homo sapiens (human) & $\begin{array}{l}\text { CLDN1, EGFR, EIF2AK2, IFIT1, OAS1, OAS2, PPP2R2B, } \\
\text { PPP2R2C, STAT1 }\end{array}$ \\
\hline hsa05168 & Herpes simplex infection-Homo sapiens (human) & $\begin{array}{l}\text { CCL2, EIF2AK2, FAS, IFIH1, IFIT1, IFNG, IL12A, IL15, } \\
\text { OAS1, OAS2, STAT1 }\end{array}$ \\
\hline hsa05142 & $\begin{array}{l}\text { Chagas disease (American } \\
\text { trypanosomiasis)-Homo sapiens (human) }\end{array}$ & C1QB, CCL2, FAS, IFNG, IL12A, PPP2R2B, PPP2R2C \\
\hline hsa04940 & Type I diabetes mellitus-Homo sapiens (human) & FAS, GAD1, IFNG, IL12A \\
\hline hsa05144 & Malaria-Homo sapiens (human) & CCL2, IFNG, IL12A, KLRK1 \\
\hline hsa05412 & $\begin{array}{l}\text { Arrhythmogenic right ventricular cardiomyopathy } \\
\text { (ARVC)-Homo sapiens (human) }\end{array}$ & CACNA2D1, DSG2, DSP, ITGAV, ITGB8 \\
\hline hsa04062 & $\begin{array}{l}\text { Chemokine signaling pathway-Homo sapiens } \\
\text { (human) }\end{array}$ & $\begin{array}{l}\text { CCL2, CCL4, CCR8, CXCL10, CXCL2, CXCL3, CXCL6, } \\
\text { CXCR6, STAT1 }\end{array}$ \\
\hline hsa04080 & $\begin{array}{l}\text { Neuroactive ligand-receptor interaction- } \\
\text { Homo sapiens (human) }\end{array}$ & $\begin{array}{l}\text { C3AR1, CHRNA7, CHRNB4, EDN1, GABRE, GAL, GHR, } \\
\text { GRIN2A, GZMA, LGR5, LHCGR, PPYR1, SSTR2 }\end{array}$ \\
\hline hsa04066 & HIF-1 signaling pathway-Homo sapiens (human) & ANGPT2, EDN1, EGFR, IFNG, NOX1, TF \\
\hline hsa05143 & African trypanosomiasis-Homo sapiens (human) & FAS, IFNG, IL12A \\
\hline hsa05410 & $\begin{array}{l}\text { Hypertrophic cardiomyopathy (HCM)- } \\
\text { Homo sapiens (human) }\end{array}$ & CACNA2D1, EDN1, ITGAV, ITGB8, TPM1 \\
\hline hsa04512 & ECM-receptor interaction-Homo sapiens (human) & COL4A5, ITGAV, ITGB8, LAMA3, LAMB3 \\
\hline hsa05132 & Salmonella infection-Homo sapiens (human) & CCL4, CXCL2, CXCL3, DYNC1I1, IFNG \\
\hline
\end{tabular}

DEGs, differentially expressed genes.

genes) were obtained via pathway analysis. Among the 31 signaling pathways corresponding to DE mRNAs, each included a different number of DEGs associated with the pathway identifier. The most significant enrichment pathway was upregulated and included 20 targeted genes associated with 'cytokine-cytokine receptor interaction-Homo sapiens (human)'. The least significantly enriched pathways were downregulated and only included two target genes; the target genes, SULT2B1 and SUOX, were involved in 'Sulfur Metabolism-Homo sapiens (human)', while the CTSG and MME genes were identified within the 'Renin-angiotensin system-Homo sapiens (human)'. In summary, 221 potential target genes are closely associated with 31 key signaling pathways in NPC (Tables I and II).

Prediction of potential target miRNAs of DE circRNAs. All DE circRNAs were annotated in detail with their respective circRNA-miRNA interaction network information (Table SVI). A total of five miRNA response elements for each DE circRNA were predicted from the results of the circRNA microarray (Table III).

Prediction of potential targets of DE circRNAs. In combination with the IncRNA, mRNA and circRNA microarray datasets, the original results of the overlap analysis were exported (Table SIX). Upon induction, 23 DE circRNAs (16 upregulated and 7 downregulated) and their associated target genes (37 miRNAs and 50 mRNAs) were selected. A regulatory network, including 37 circRNA-miRNA interactions and 50 miRNA-mRNA interactions, was then constructed (Table VI and Fig. 4), in which 14 circRNA-miRNA-mRNA regulatory modules were identified (Fig. 5). According to the data, there is no one-to-one correspondence between circRNA and its target genes. For example, hsa_circRNA_104405 
Table II. Key signaling pathways associated with downregulated DEGs.

\begin{tabular}{|c|c|c|}
\hline Pathway ID & Definition & DEGs \\
\hline hsa04640 & Hematopoietic cell lineage-Homo sapiens (human) & $\begin{array}{l}\text { CD19, CD1C, CD22, CD37, CR1, CR2, FCER2, MME, } \\
\text { MS4A1 }\end{array}$ \\
\hline hsa04080 & $\begin{array}{l}\text { Neuroactive ligand-receptor interaction- } \\
\text { Homo sapiens (human) }\end{array}$ & $\begin{array}{l}\text { ADRA2A, CNR2, CTSG, EDN3, GALR2, GPR77, } \\
\text { GRM5, HTR2A, LEP, MC1R, P2RX5, PRSS1, PRSS3, } \\
\text { S1PR4, SCT, TAC4 }\end{array}$ \\
\hline hsa04662 & $\begin{array}{l}\text { B cell receptor signaling pathway-Homo sapiens } \\
\text { (human) }\end{array}$ & CD19, CD22, CD72, CD79A, CD79B, CR2, VAV3 \\
\hline hsa05217 & Basal cell carcinoma - Homo sapiens (human) & GLI1, PTCH1, TCF7, WNT16, WNT9A \\
\hline hsa05340 & $\begin{array}{l}\text { Primary immunodeficiency-Homo sapiens } \\
\text { (human) }\end{array}$ & CD19, CD40LG, CD79A, TNFRSF13C \\
\hline hsa04340 & $\begin{array}{l}\text { Hedgehog signaling pathway-Homo sapiens } \\
\text { (human) }\end{array}$ & GLI1, PTCH1, WNT16, WNT9A \\
\hline hsa04064 & $\begin{array}{l}\text { NF-kappa B signaling pathway-Homo sapiens } \\
\text { (human) }\end{array}$ & CCL19, CCL21, CD40LG, CXCL12, TNFRSF13C \\
\hline hsa00920 & Sulfur metabolism-Homo sapiens (human) & SULT2B1, SUOX \\
\hline hsa04614 & Renin-angiotensin system-Homo sapiens (human) & CTSG, MME \\
\hline hsa04060 & $\begin{array}{l}\text { Cytokine-cytokine receptor interaction- } \\
\text { Homo sapiens (human) }\end{array}$ & $\begin{array}{l}\text { AMHR2, CCL17, CCL19, CCL21, CD40LG, CXCL12, } \\
\text { LEP, TNFRSF10D, TNFRSF13C }\end{array}$ \\
\hline hsa04062 & $\begin{array}{l}\text { Chemokine signaling pathway-Homo sapiens } \\
\text { (human) }\end{array}$ & $\begin{array}{l}\text { CCL17, CCL19, CCL21, CXCL12, GNG7, RASGRP2, } \\
\text { VAV3 }\end{array}$ \\
\hline hsa04672 & $\begin{array}{l}\text { Intestinal immune network for IgA } \\
\text { production-Homo sapiens (human) }\end{array}$ & CD40LG, CXCL12, TNFRSF13C \\
\hline hsa05030 & Cocaine addiction-Homo sapiens (human) & CDK5R1, DLG4, FOSB \\
\hline
\end{tabular}

DEGs, differentially expressed genes.

is associated with 2 target miRNAs (hsa-miR-122-5p and hsa-miR-205-5p), while hsa-miR-122 is associated with 1 target mRNA (RIMS1) and hsa-miR-205-5p is associated with 3 target mRNAs (CENPF, FRK and SCD5).

\section{Discussion}

NPC is a type of head and neck cancer with a high incidence and poor overall survival rate, particularly in the endemic regions of Southeast Asia (4). Although a clear understanding of its etiology is yet to be determined, NPC is widely suspected to be the result of both genetic susceptibility, exposure to certain environmental factors or Epstein-Barr virus infection $(1,2)$. Genome-wide association and regulatory ncRNA studies may improve understanding of the etiological and essential molecular mechanisms underpinning NPC progres$\operatorname{sion}(14,16,17)$.

The non-coding regions of the human genome have been closely associated with the biological processes of disease (6). Furthermore, it has been demonstrated that IncRNAs, miRNAs and circRNAs all regulate the physiological and pathological processes of numerous types of cancer, and that these regulatory ncRNAs can affect the functions of their target mRNAs $(11,17-19)$. It has been reported that ncRNA molecules influence tumorigenesis and tumor progression by forming regulatory networks with their target genes (10), which corresponds with the multi-gene and multi-step regulation of tumor development.

Certain studies have investigated the ncRNA regulatory networks that influence the occurrence and development of various types of tumor, including NPC $(10,11,13,20)$. Therefore, in order to delineate an NPC-specific regulatory gene network containing ncRNAs in $>10,000$ human genes, the comprehensive identification of NPC-associated DE ncRNAs and their targets represents the initial step in establishing this network. To the best of our knowledge, the present study is the first to simultaneously screen and predict the possible target genes of DE ncRNAs (lncRNAs, miRNAs and circRNAs) using three sets of high-throughput microarray data based on transcriptome profiling of NPC tissues. The results constitute a foundation for subsequent comprehensive studies into the regulatory network behind the molecular pathogenesis of NPC.

In the present study, $>100$ DE IncRNAs and mRNAs were identified in NPC tissues. Subsequently, the NPC-associated nearby coding genes that may represent targets of DE lncRNAs were predicted via bioinformatics analysis, and their associations between DE IncRNAs, nearby coding genes and genome coordinates were also evaluated. Pathway analysis was conducted to determine the biological function of the selected DE mRNAs in NPC pathogenesis, and to predict the essential genes regulating various NPC-associated signaling pathways. 

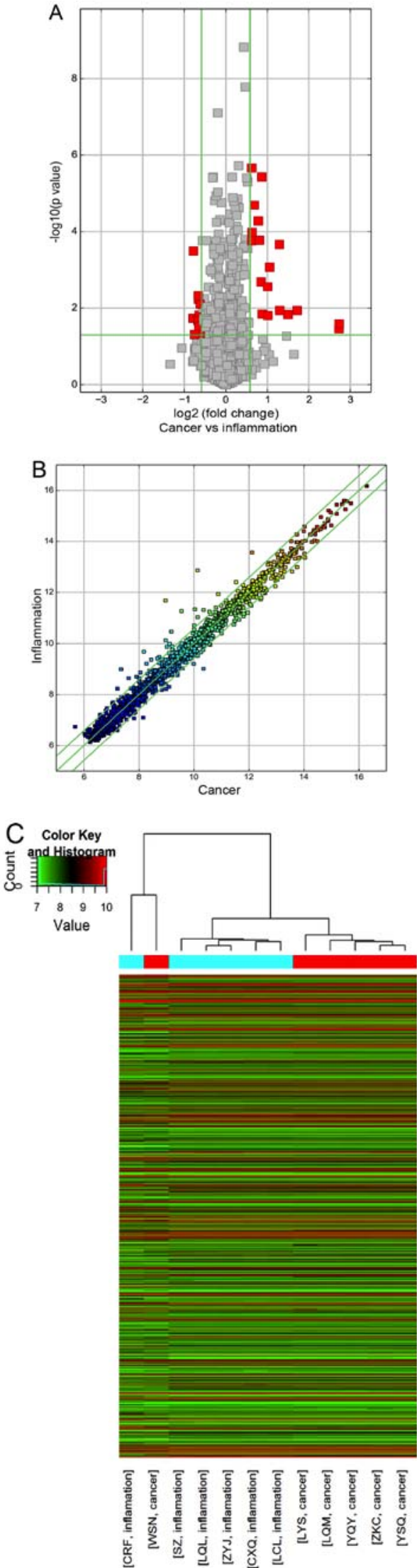

Figure 2. Bioinformatics analysis of differentially expressed circRNAs in NPC and CNP tissues. (A) Volcano plot demonstrating the distribution of circRNAs (B) Scatter plot exhibiting variation in circRNA expression. (C) Heatmap clustering indicating a distinguishable association between the circRNA expression patterns of certain samples. circRNAs expression levels are indicated as follows; Red, high expression and green, low expression. circRNA, circular RNA; NPC, nasopharyngeal carcinoma; CNP, chronic nasopharyngitis.
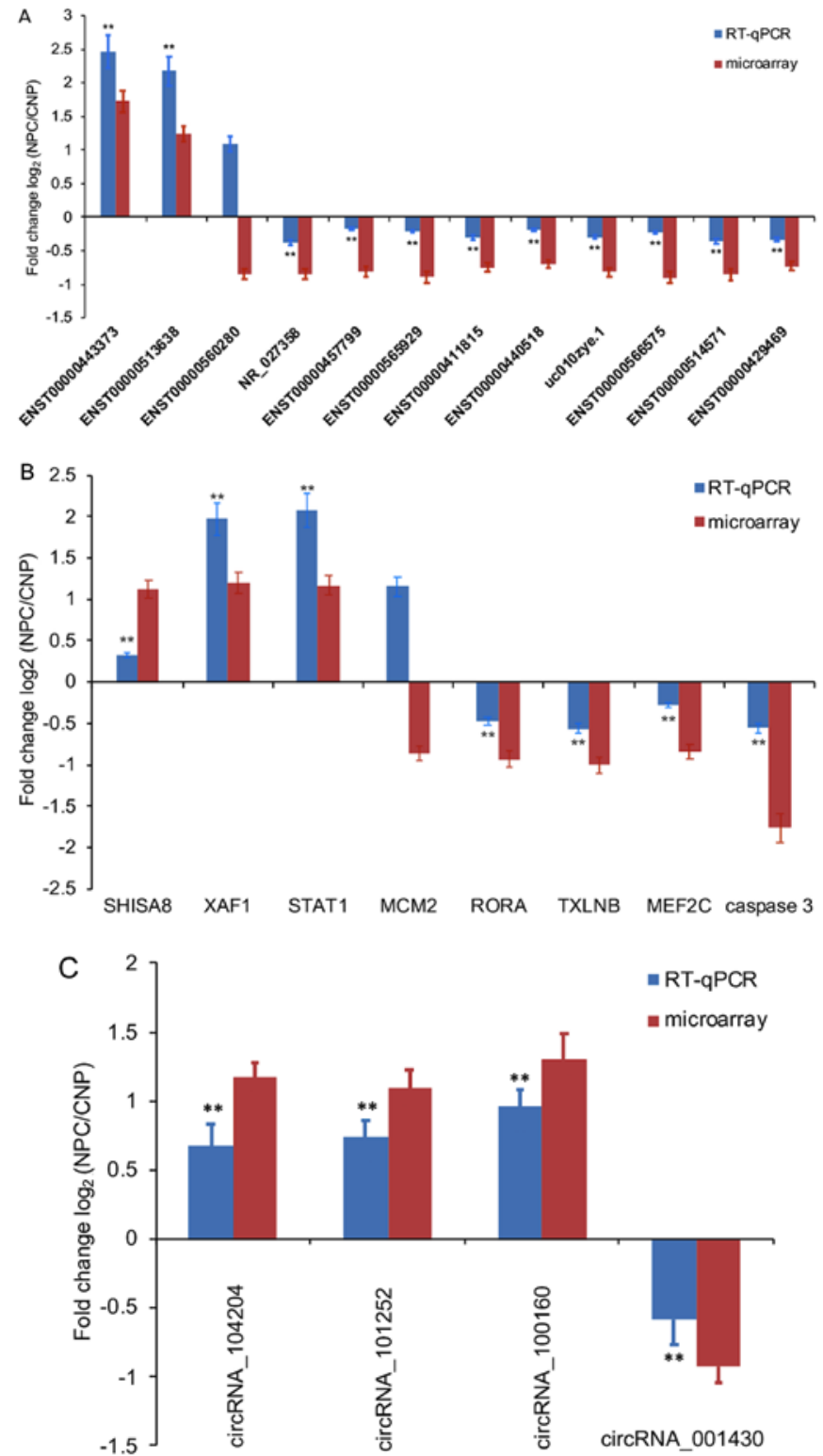

Figure 3. Validation of selected (A) lncRNAs, (B) mRNAs and (C) circRNAs based on microarray and RT-qPCR data. Column heights represent the fold changes ( $\log 2$ transformed) between NPC and CNP tissues, determined using RT-qPCR and microarray data. The validation results indicated a positive association between the RT-qPCR and microarray data. ${ }^{* *} \mathrm{P}<0.01$ vs. microarray. lncRNA, long non-coding RNA; circRNA, circular RNA; RT-qPCR, reverse transcription-quantitative PCR; NPC, nasopharyngeal carcinoma; CNP, chronic nasopharyngitis.

Additional target genes of DE lncRNAs were identified from 31 significantly enriched signaling pathways associated with NPC. The results suggest that aberrantly expressed lncRNAs may influence NPC development and progression through certain mechanisms, such as the interaction between a DE lncRNA and its adjacent protein-coding gene, or via interaction with its target gene in the corresponding signaling pathway. Thus, lncRNA-mRNA networks may serve an important role in the transcriptional regulation of NPC.

Pathway analysis demonstrated that 31 signaling pathways were associated with DEGs, including 18 pathways associated with upregulated, and 13 associated with downregulated genes; three of these pathways ("cytokine-cytokine receptor 


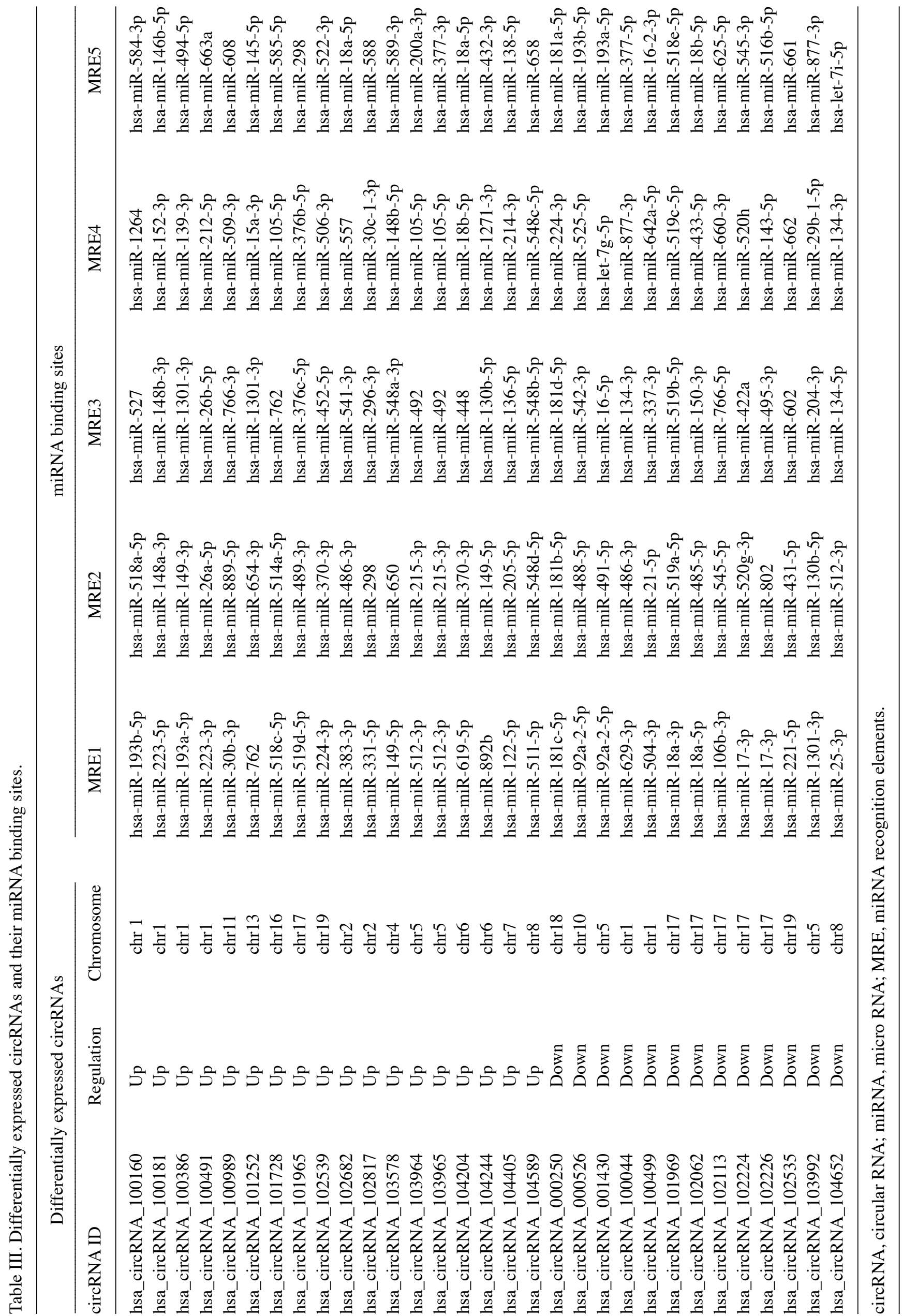


Table IV. Nearby coding genes for long intergenic non-coding RNAs.

\begin{tabular}{|c|c|c|c|c|}
\hline Sequence name & Gene symbol & Genomic location & GR & Nearby gene \\
\hline AW833912 & & Chr 3: 172556888-172557496 & $\mathrm{D}$ & NM_018098 \\
\hline BF108976 & & Chr 2: $192068946-192069452$ & $\mathrm{D}$ & NM_007315 \\
\hline BG953017R & & Chr 4: $184736396-184736595$ & $\mathrm{U}$ & ENST00000296741 \\
\hline ENST00000400353 & AP000569.8 & Chr 21: $35303517-35343487$ & $\mathrm{U}$ & NM_001001132 \\
\hline ENST00000411844 & KIAA0664L3 & Chr 16: 3171560031717339 & $\mathrm{U}$ & ENST00000389202 \\
\hline ENST00000412797 & RP11-70P17.1 & Chr 1: 25907968-25916847 & $\mathrm{D}$ & NM_024037 \\
\hline ENST00000413991 & AC073257.2 & Chr 2: $121300484-121301902$ & $\mathrm{U}$ & NM_005270 \\
\hline ENST00000420672 & AC009948.5 & Chr 2: $179278665-179295551$ & $\mathrm{U}$ & NM_145739 \\
\hline ENST00000425214 & CCDC144B & Chr 17: $18494172-18507053$ & $\mathrm{U}$ & NM_016078 \\
\hline ENST00000431729 & RP11-191N8.2 & Chr 1: 222001007-222014008 & $\mathrm{D}$ & NM_144729 \\
\hline ENST00000434893 & GUSBP11 & Chr 22: 23995356-24029101 & $\mathrm{U}$ & NM_013378 \\
\hline ENST00000438082 & RP11-57C13.6 & Chr 10: 89367741-89419036 & $\mathrm{D}$ & NM_004670 \\
\hline ENST00000439051 & RP11-57C13.6 & Chr 10: 89367768-89419036 & $\mathrm{D}$ & NM_004670 \\
\hline ENST00000439472 & TTTY10 & Chr Y: 22669139-22680293 & $\mathrm{U}$ & NM_001039567 \\
\hline ENST00000440357 & RP4-738P15.1 & Chr 20: $25124000-25129876$ & $\mathrm{D}$ & ENST00000480798 \\
\hline ENST00000441287 & AC011193.1 & Chr 17: $32806352-32806976$ & $\mathrm{U}$ & NM_002982 \\
\hline ENST00000442583 & CCDC144B & Chr 17: $18491592-18509704$ & $\mathrm{U}$ & NM_016078 \\
\hline ENST00000449023 & SRGAP3-AS4 & Chr 3: 9298442-9299191 & $\mathrm{U}$ & NM_014850 \\
\hline ENST00000457217 & RP11-222A5.1 & Chr 1: 175846478-175849604 & $\mathrm{U}$ & NM_003285 \\
\hline ENST00000483245 & RP11-202A13.1 & Chr 3: 133774099-133776492 & $\mathrm{D}$ & NM_001063 \\
\hline ENST00000486295 & EGFEM1P & Chr 3: $168538977-168547319$ & $\mathrm{D}$ & ENST00000264674 \\
\hline ENST00000514571 & CTC-454M9.1 & Chr 5: 88261691-88464485 & $\mathrm{U}$ & NM_001193347 \\
\hline ENST00000520323 & CTB-11I22.2 & Chr 5: $158654722-158672135$ & $\mathrm{U}$ & NM_024007 \\
\hline ENST00000520840 & RP11-875011.3 & Chr 8: 22928889-22932001 & $\mathrm{U}$ & ENST00000312584 \\
\hline ENST00000536112 & RP11-81H14.2 & Chr 12: 68825634-68826434 & $\mathrm{D}$ & NM_000619 \\
\hline ENST00000537192 & RP11-1038A11.3 & Chr 12: $5399645-5487520$ & $\mathrm{D}$ & NM_002527 \\
\hline ENST00000538430 & RP11-1038A11.1 & Chr 12: $5497754-5515817$ & $\mathrm{D}$ & NM_002527 \\
\hline ENST00000539404 & RP11-81H14.2 & Chr 12: 68726727-68797580 & $\mathrm{D}$ & NM_000619 \\
\hline ENST00000541707 & RP11-81H14.2 & Chr 12: 68726667-68729561 & $\mathrm{D}$ & NM_000619 \\
\hline ENST00000544591 & RP11-291B21.2 & Chr 12: 10705961-10710648 & $\mathrm{U}$ & NM_007333 \\
\hline ENST00000544842 & RP11-319E16.2 & Chr 12: $5425126-5428513$ & $\mathrm{D}$ & NM_002527 \\
\hline ENST00000546086 & RP11-81H14.2 & Chr 12: 68727032-68835996 & $\mathrm{D}$ & NM_000619 \\
\hline ENST00000546968 & RP11-44N21.1 & Chr 14: 105561527-105565341 & $\mathrm{U}$ & NM_138790 \\
\hline ENST00000548846 & RP3-473L9.4 & Chr 12: $111834638-111841111$ & $\mathrm{D}$ & NM_001136538 \\
\hline ENST00000549710 & RP11-498M15.1 & Chr 12: $72102950-72104154$ & $\mathrm{D}$ & NM_003667 \\
\hline ENST00000552154 & RP11-554D14.7 & Chr 12: $108226634-108228807$ & $\mathrm{D}$ & ENST00000342331 \\
\hline ENST00000556624 & RP11-219E7.1 & Chr 14: 21252046-21252452 & $\mathrm{D}$ & ENST00000298687 \\
\hline ENST00000558147 & LINC00277 & Chr 15; 69373189-69383734 & $\mathrm{U}$ & ENST00000310673 \\
\hline ENST00000558419 & CTD-2008A1.1 & Chr 15: 45118737-45119292 & $\mathrm{D}$ & NM_003104 \\
\hline ENST00000559914 & LINC00277 & Chr 15: 69365277-69367206 & $\mathrm{U}$ & ENST00000310673 \\
\hline ENST00000561384 & CTD-2008A1.2 & Chr 15: 45119397-45176892 & $\mathrm{U}$ & NM_003104 \\
\hline ENST00000562834 & RP3-523K23.2 & Chr 6: 54807964-54809897 & $\mathrm{U}$ & NM_001010872 \\
\hline ENST00000563852 & RP11-506G7.1 & Chr 17: 41020507-41025481 & $\mathrm{D}$ & NM_007299 \\
\hline ENST00000564832 & RP11-531A24.3 & Chr 8: 73859384-73862680 & $\mathrm{D}$ & NM_001243237 \\
\hline ENST00000566575 & CTA-250D10.23 & Chr 22: $42318026-42319104$ & $\mathrm{D}$ & NM_001207020 \\
\hline ENST00000568337 & RP11-160C18.2 & Chr 15: 79021382-79026298 & $\mathrm{U}$ & NM_000750 \\
\hline ENST00000569215 & RP11-609N14.1 & Chr 16: 10445296-10446609 & $\mathrm{D}$ & NM_001134407 \\
\hline ENST00000569655 & RP11-143K11.1 & Chr 17: 71171621-71172772 & $\mathrm{U}$ & NM_001050 \\
\hline ENST00000569892 & RP11-114H24.3 & Chr 15: 78246416-78255996 & $\mathrm{U}$ & NM_015162 \\
\hline ENST00000575693 & LA16c-325D7.2 & Chr 16: 2916348-2917619 & $\mathrm{U}$ & NM_024507 \\
\hline ENST00000577807 & RP11-599B13.3 & Chr 17: 7959542-7960939 & $\mathrm{D}$ & NM_001039131 \\
\hline HMlincRNA791- & HMlincRNA791 & Chr 18: $52298998-52308760$ & $\mathrm{U}$ & NM_001143829 \\
\hline
\end{tabular}


Table IV. Continued.

\begin{tabular}{|c|c|c|c|c|}
\hline Sequence name & Gene symbol & Genomic location & GR & Nearby gene \\
\hline HMlincRNA963+ & HMlincRNA963 & Chr 3: 168554930-168560248 & $\mathrm{D}$ & ENST00000264674 \\
\hline NR_024475 & LOC100216001 & Chr 10: 4692376-4720262 & $\mathrm{U}$ & NM_001353 \\
\hline NR_026878 & FOXD2-AS1 & Chr 1: 47897806-47900313 & $\mathrm{D}$ & ENST00000337817 \\
\hline NR_027994 & NHEG1 & Chr 6: $137303295-137314368$ & $\mathrm{U}$ & NM_181310 \\
\hline NR_038293 & LOC100507173 & Chr 6: 27661813-27678001 & $\mathrm{D}$ & ENST00000331442 \\
\hline NR_040109 & LOC100505495 & Chr 19: 41960073-42006554 & $\mathrm{U}$ & NM_006890 \\
\hline TCONS_00001315 & XLOC_000595 & Chr 1: 227976987-227979782 & $\mathrm{D}$ & NM_003395 \\
\hline TCONS_00001451 & XLOC_000781 & Chr 1: 35081179-35083207 & $\mathrm{U}$ & NM_005268 \\
\hline TCONS_00005258 & XLOC_002368 & Chr 2: 160780449-160792478 & $\mathrm{U}$ & NM_001007267 \\
\hline TCONS_00005268 & XLOC_002383 & Chr 2: 169197716-169198115 & $\mathrm{U}$ & NM_203463 \\
\hline TCONS_00006514 & XLOC_003131 & Chr 3: 54048256-54065456 & $\mathrm{D}$ & NM_018397 \\
\hline TCONS_00008529 & XLOC_004016 & Chr 4: 90459366-90472707 & $\mathrm{U}$ & ENST00000420646 \\
\hline TCONS_00009933 & XLOC_004361 & Chr 5: 42922835-42924839 & $\mathrm{U}$ & NM_000163 \\
\hline TCONS_00010742 & XLOC_004475 & Chr 5: 92906525-92909378 & $\mathrm{D}$ & NM_005654 \\
\hline TCONS_00011633 & XLOC_005123 & Chr 6: 1489677-1490173 & $\mathrm{U}$ & NM_033260 \\
\hline TCONS_00011758 & XLOC_005220 & Chr 6: 27677988-27680876 & $\mathrm{D}$ & ENST00000331442 \\
\hline TCONS_00012442 & XLOC_005214 & Chr 6: 26674955-26677930 & $\mathrm{U}$ & NM_001732 \\
\hline TCONS_00012443 & XLOC_005214 & Chr 6: 26675224-26688063 & $\mathrm{U}$ & NM_001732 \\
\hline TCONS_00014617 & XLOC_006712 & Chr 8: 11500332-11506826 & $\mathrm{U}$ & NM_001715 \\
\hline TCONS_00014681 & XLOC_006779 & Chr 8: 39891375-39891902 & $\mathrm{U}$ & NM_001464 \\
\hline TCONS_00017282 & XLOC_008100 & Chr X: 2484083-2488088 & $\mathrm{U}$ & NM_001141919 \\
\hline TCONS_00017293 & XLOC_008116 & Chr X: 13405670-13437996 & $\mathrm{U}$ & NM_001167890 \\
\hline TCONS_00018417 & XLOC_008704 & Chr 10: 4790106-4806336 & $\mathrm{U}$ & NM_001353 \\
\hline TCONS_00021032 & XLOC_009637 & Chr 12: 7491433-7494514 & $\mathrm{U}$ & NM_031491 \\
\hline TCONS_00021064 & XLOC_009662 & Chr 12: 10725616-10727581 & $\mathrm{U}$ & NM_007333 \\
\hline TCONS_00029036 & XLOC_013955 & Chr 21: 44232379-44237997 & $\mathrm{U}$ & NM_001001568 \\
\hline TCONS_00029753 & XLOC_014147 & Chr 22: 18848963-18851914 & $\mathrm{U}$ & NM_017414 \\
\hline TCONS_00029855 & XLOC_014297 & Chr 22: 19543858-19552723 & $\mathrm{D}$ & NM_001178010 \\
\hline uc001yfd.1 & BX247990 & Chr 14: 96181819-96223116 & $\mathrm{D}$ & NM_001252507 \\
\hline uc002ebp.1 & TRIM72 & Chr 16: 31237192-31237830 & $\mathrm{D}$ & ENST00000389202 \\
\hline uc002iby. 2 & LOC388387 & Chr 17: 41026690-41050751 & $\mathrm{D}$ & NM_001158 \\
\hline uc002nbr.3 & UCA1 & Chr 19: 15939756-15946230 & $\mathrm{D}$ & ENST00000344824 \\
\hline uc002zbk.2 & ВC041455 & Chr 21: 44019513-44035168 & $\mathrm{U}$ & NM_001001568 \\
\hline .uc002zob.1 & GGT3P & Chr 22: 18761201-18792992 & $\mathrm{D}$ & NM_017414 \\
\hline uc003fif.1 & AK127557 & Chr 3: 172308502-172312373 & $\mathrm{D}$ & ENST00000241261 \\
\hline uc003ihb.3 & ВC042378 & Chr 4: 134114523-134115760 & $\mathrm{U}$ & ENST00000264360 \\
\hline uc003qhh.4 & NHEG1 & Chr 6: $137303295-137314368$ & $\mathrm{U}$ & NM_181310 \\
\hline uc010jbc.2 & FLJ42709 & Chr 5: 92877577-92916738 & $\mathrm{U}$ & NM_005654 \\
\hline uc010vdm.1 & RRN3P2 & Chr 16: 29086162-29107582 & $\mathrm{U}$ & NM_001178098 \\
\hline
\end{tabular}

Chr, chromosome; GR, genome relationship; U, upstream; D, downstream.

interaction', 'chemokine signaling pathway' and 'neuroactive ligand-receptor interaction') were simultaneously associated with upregulated and downregulated signaling pathways. Of the 74 target genes associated with the above three pathways, 14 genes (LEP, CCL17, CCL19, CCL21, CXCL12, CCL2, CCL4, CCR8, CXCL10, CXCL2, CXCL3, CXCL6, CXCR6 and GHR) were separately involved in $\geq 2$ of these pathways. The functions of these target genes were associated with the following biological processes: 'Signal transduction', 'cell adhesion and migration', 'cell proliferation', ‘inflammatory cell infiltration', 'angiogenesis' and 'immunoregulation'. Additionally, various pathways and target genes were associated with the development and progression of several other human cancer types (21-23). The present results indicate that the three aforementioned pathways, and 14 identified genes, may represent key regulators of NPC tumorigenesis.

Additionally, multiple DE circRNAs associated with $\mathrm{NPC}$, and their target miRNAs/mRNAs, were investigated 
Table V. Nearby coding genes for enhancer long non-coding RNAs.

\begin{tabular}{lllll}
\hline Sequence name & Gene symbol & \multicolumn{1}{c}{ Genomic location } & GR & Nearby gene \\
\hline ENST00000400353 & AP000569.8 & Chr 21: 35303517-35343487 & U & NM_001001132 \\
ENST00000411844 & KIAA0664L3 & Chr 16: 31715600-31717339 & U & ENST00000389202 \\
ENST00000412797 & RP11-70P17.1 & Chr 1: 25907968-25916847 & D & NM_024037 \\
ENST00000431729 & RP11-191N8.2 & Chr 1: 222001007-222014008 & D & NM_144729 \\
ENST00000440357 & RP4-738P15.1 & Chr 20: 25124000-25129876 & D & ENST00000480798 \\
ENST00000440357 & RP4-738P15.1 & Chr 20: 25124000-25129876 & U & NM_021067 \\
ENST00000441287 & AC011193.1 & Chr 17: 32806352-32806976 & U & NM_002982 \\
NR_024475 & LOC100216001 & Chr 10: 4692376-4720262 & U & NM_001353 \\
NR_026878 & FOXD2-AS1 & Chr 1: 47897806-47900313 & D & ENST00000337817 \\
NR_027994 & NHEG1 & Chr 6: 137303295-137314368 & U & NM_181310 \\
uc003qhh.4 & NHEG1 & Chr 6: 137303295-137314368 & U & NM_181310
\end{tabular}

Chr, chromosome; GR, genome relationship; U, upstream; D, downstream.

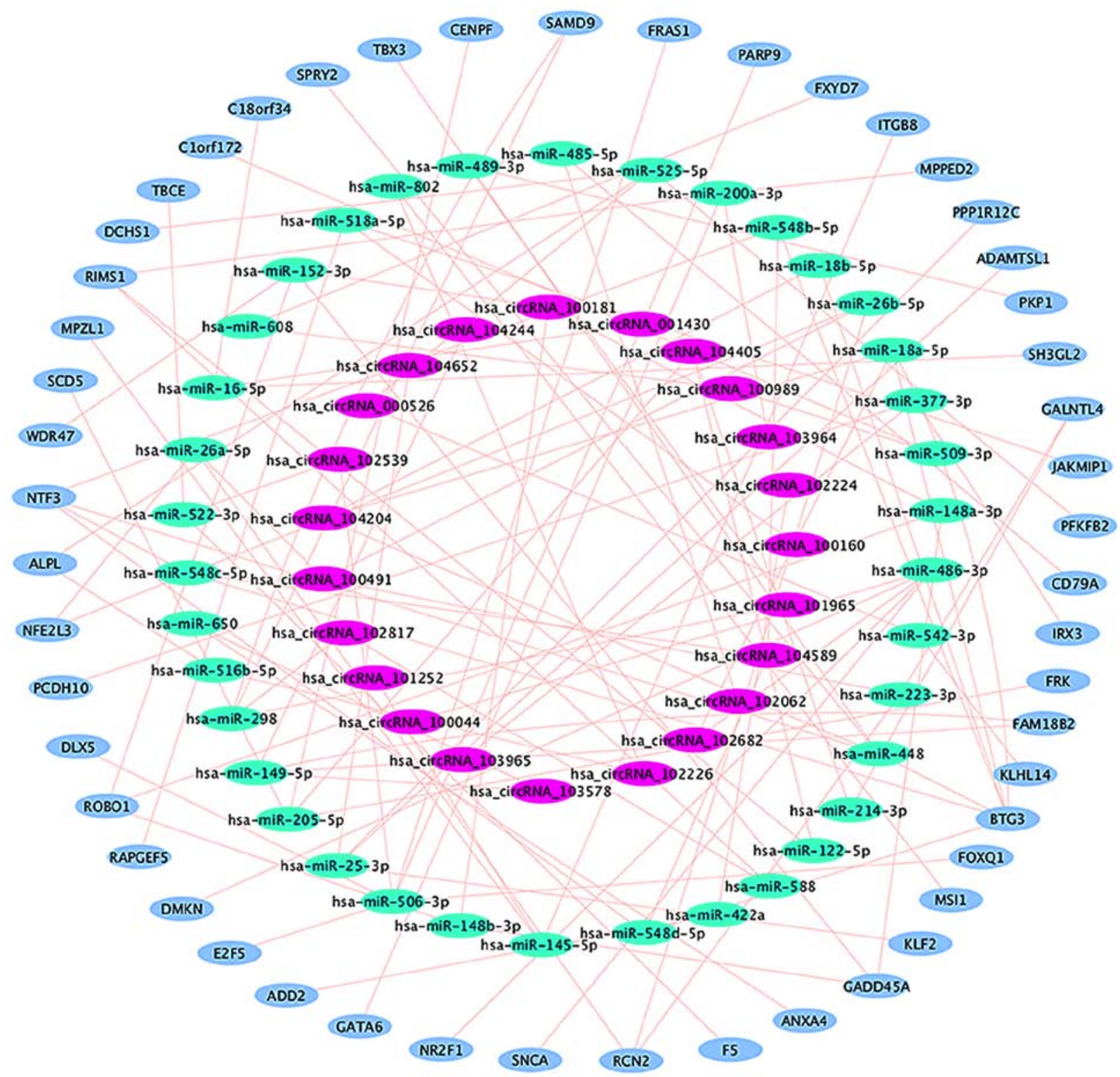

Figure 4. circRNA-miRNA-mRNA regulatory network in nasopharyngeal carcinoma. Green circle, miRNA; pink circle, circRNA; light-blue circle, mRNA. circRNA, circular RNA; miRNA, micro RNA. 
Table VI. Regulatory network components of differentially expressed circRNAs in nasopharyngeal carcinoma.

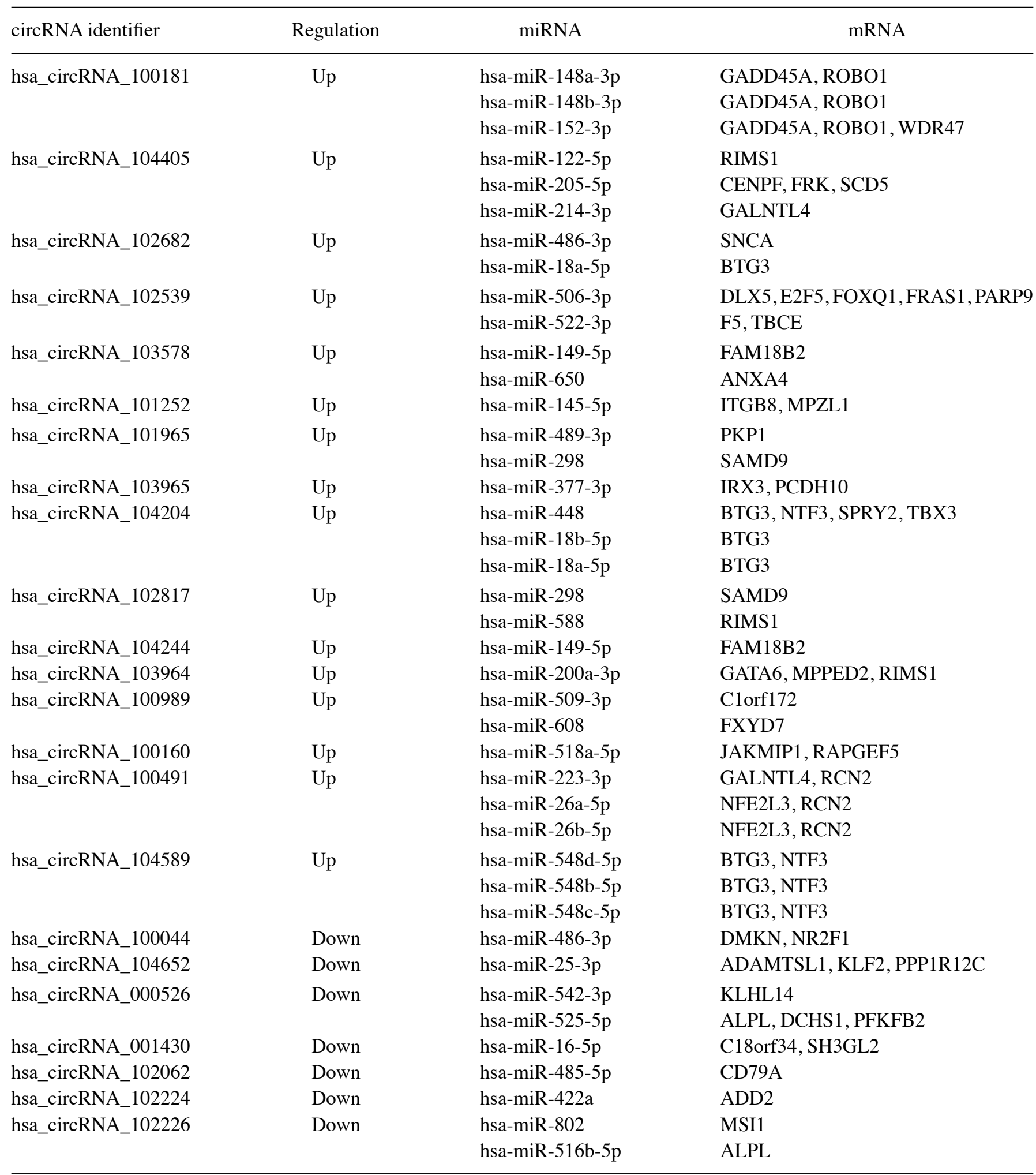

circRNA, circular RNA; miRNA/miR, micro RNA.

alongside their corresponding association and genome mapping. However, thus far, no detailed reports are available on the association between circRNAs and their targets in NPC. The present study identified regulatory circRNA-miRNA-mRNA networks in NPC, which contained different modules consisting of relevant target genes. The current results indicate that aberrantly expressed circRNAs may influence different pathophysiological mechanisms of NPC via interaction with miRNAs and mRNAs, and also that the circRNA-miRNA-mRNA motifs serve a key regulatory 


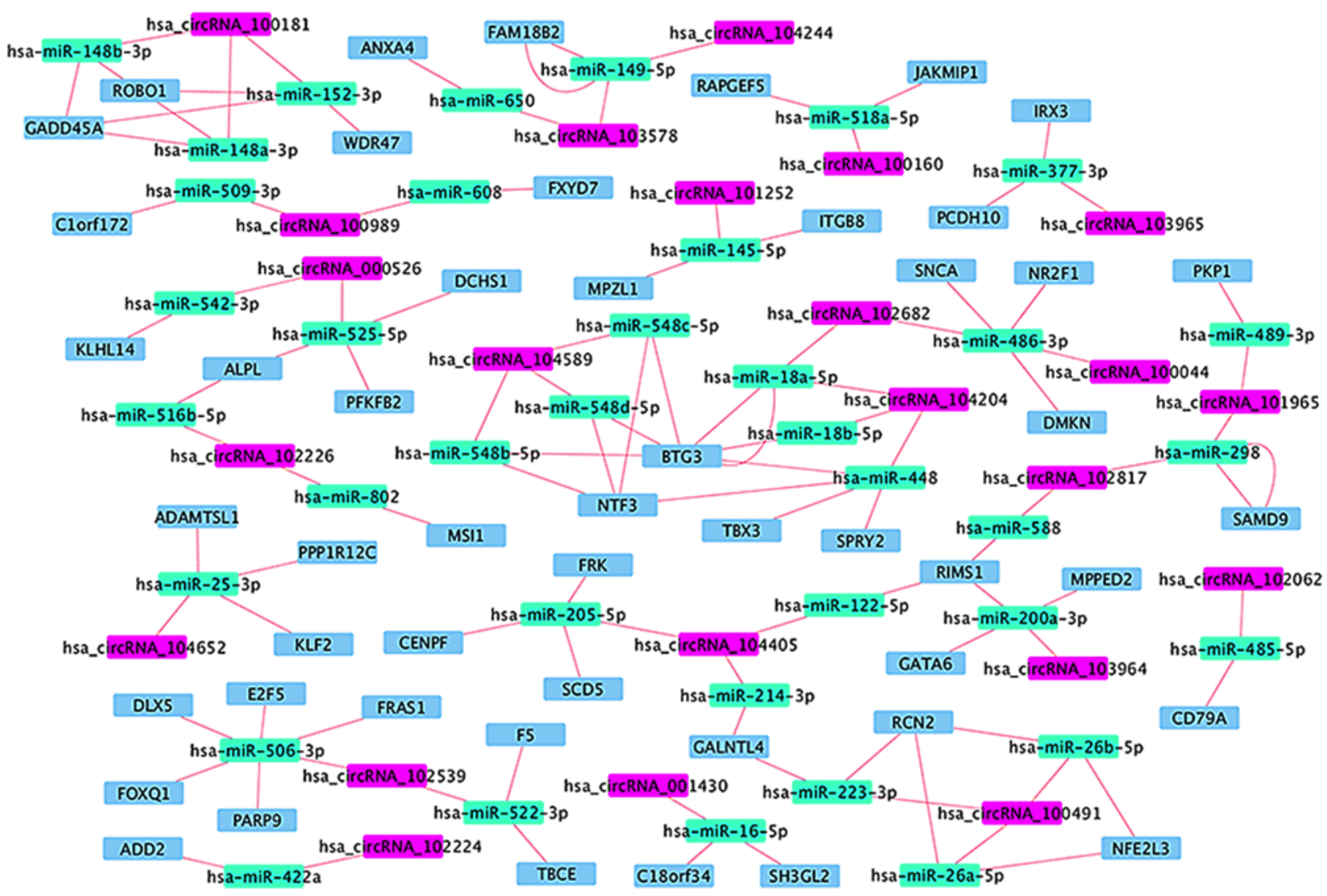

Figure 5. circRNA-miRNA-mRNA regulatory modules in nasopharyngeal carcinoma. Green rectangle, miRNA; pink rectangle, circRNA; light-blue rectangle, mRNA. circRNA, circular RNA; miRNA, micro RNA.

function in NPC. Taken together, the present data indicate that IncRNAs do not serve an isolated role, but target the mRNAs of various other genes, and influence other associated genes involved in the tumorigenesis and progression of NPC, by forming regulatory networks.

In the circRNA-miRNA-mRNA network, 50 mRNAs were identified as the final functional genes. According to the National Center for Biotechnology Information gene database, the functions of these target genes were associated with the following physiological and pathological mechanisms: 'Environmental stress', 'cell motility and migration', 'cytoskeleton', 'antiproliferative activity', 'regulation of voltage-gated calcium channels', 'cell proliferation and apoptosis', 'desmosome formation', 'annexin', 'B lymphocyte antigen receptor' 'bimodal regulator of epidermal growth factor receptor and mitogen-activated protein kinase signaling', 'extracellular matrix protein' and 'chromosome segregation'. Notably, previous studies have revealed that certain identified target genes are associated with cancer cell proliferation and metastasis $(24,25)$, and thus serve important roles in NPC development and progression (26-28).

Overall, the present study simultaneously identified DE lncRNAs, circRNAs and mRNAs between NPC and CNP tissues via the integrated analysis of three transcriptome profiling datasets. Furthermore, potential target genes for these DE ncRNAs, and key signaling pathways associated with NPC, were identified using bioinformatics analysis. Finally, possible regulatory networks comprised of different modules in NPC were predicted and constructed. The present study serves to evaluate the association between these genes and NPC at the RNA transcriptome level. It also provides novel information to elucidate the molecular pathogenesis of NPC from a networking perspective. In further studies, the biological functions of these regulatory networks in NPC should be verified.

\section{Acknowledgements}

Not applicable.

\section{Funding}

The present study was funded by the Natural Science Fund of Fujian Province, China (grant no. 2017J01374).

\section{Availability of data and materials}

All data generated or analyzed during this study are included in this published article.

\section{Authors' contributions}

YFD designed the study. YFD and CSY revised the manuscript. DNZ, CSY and QQY performed the research, collected and analyzed the data, and wrote the manuscript. All authors read and approved the final manuscript. 


\section{Ethics approval and consent to participate}

The present study was ethically approved by the Medical Ethics Committee of Zhongshan Hospital, Xiamen University (Xiamen, China), and written informed consent was obtained from all subjects prior to the study start.

\section{Patient consent for publication}

Not applicable.

\section{Competing interests}

The authors declare that they have no competing interests.

\section{References}

1. Tsang CM, Lui VWY, Bruce JP, Pugh TJ and Lo KW: Translational genomics of nasopharyngeal cancer. Semin Cancer Biol, Sep 12, 2019 (Epub ahead of print).

2. Chen YP, Chan ATC, Le QT, Blanchard P, Sun Y and Ma J: Nasopharyngeal carcinoma. Lancet 394: 64-80, 2019.

3. Wilusz JE and Sharp PA: A circuitous route to noncoding RNA. Science 340: 440-441, 2013.

4. Wu J and Hann SS: Functions and roles of long-non-coding RNAs in human nasopharyngeal carcinoma. Cell Physiol Biochem 45: 1191-1204, 2018

5. Anastasiadou E, Jacob LS and Slack FJ: Non-coding RNA networks in cancer. Nat Rev Cancer 18: 5-18, 2018.

6. Li LJ, Leng RX, Fan YG, Pan HF and Ye DQ: Translation of noncoding RNAs: Focus on lncRNAs, pri-miRNAs, and circRNAs. Exp Cell Res 361: 1-8, 2017.

7. Bhan A, Soleimani M and Mandal SS: Long noncoding RNA and cancer: A new paradigm. Cancer Res 77: 3965-3981, 2017.

8. Yang QQ and Deng YF: Long non-coding RNAs as novel biomarkers and therapeutic targets in head and neck cancers. Int J Clin Exp Pathol 7: 1286-1292, 2014.

9. Anfossi S, Fu X, Nagvekar R and Calin GA: MicroRNAs, regulatory messengers inside and outside cancer cells. Adv Exp Med Biol 1056: 87-108, 2018.

10. Zang J, Lu D and $\mathrm{Xu} A$ : The interaction of circRNAs and RNA binding proteins: An important part of circRNA maintenance and function. J Neurosci Res 98: 87-97, 2020.

11. Gong Z, Yang Q, Zeng Z, Zhang W, Li X, Zu X, Deng H, Chen P, Liao Q, Xiang B, et al: An integrative transcriptomic analysis reveals p53 regulated miRNA, mRNA, and lncRNA networks in nasopharyngeal carcinoma. Tumour Biol 37: 3683-3695, 2016.

12. Ma DD, Yuan LL and Lin LQ: IncRNA HOTAIR contributes to the tumorigenesis of nasopharyngeal carcinoma via up-regulating FASN. Eur Rev Med Pharmacol Sci 21: 5143-5152, 2017.

13. Zhang ZZ, Cao HC, Huang DL, Chen XF, Wan J and Zhang W: MicroRNA-200c plays an oncogenic role in nasopharyngeal carcinoma by targeting PTEN. Tumour Biol 39: 1010428317703655 2017.
14. Shuai M, Hong J, Huang D, Zhang X and Tian Y: Upregulation of circRNA_0000285 serves as a prognostic biomarker for nasopharyngeal carcinoma and is involved in radiosensitivity. Oncol Lett 16: 6495-6501, 2018.

15. Livak KJ and Schmittgen TD: Analysis of relative gene expression data using real-time quantitative PCR and the 2(-Delta Delta $\mathrm{C}(\mathrm{T})$ ) method. Methods 25: 402-408, 2001.

16. Paul P, Deka H, Malakar AK, Halder B and Chakraborty S: Nasopharyngeal carcinoma: Understanding its molecular biology at a fine scale. Eur J Cancer Prev 27: 33-41, 2018.

17. Nicolas FE: Role of ncRNAs in development, diagnosis and treatment of human cancer. Recent Pat Anticancer Drug Discov 12: 128-135, 2017.

18. Kristensen LS, Hansen TB, Venø MT and Kjems J: Circular RNAs in cancer: Opportunities and challenges in the field. Oncogene 37: 555-565, 2018.

19. Du WW, Zhang C, Yang W, Yong T, Awan FM and Yang BB: Identifying and characterizing circRNA-protein interaction. Theranostics 7: 4183-4191, 2017.

20. Liu M, Zhu K, Qian X and Li W: Identification of miRNA/mRNA-negative regulation pairs in nasopharyngeal carcinoma. Med Sci Monit 22: 2215-2234, 2016.

21. Zhang H, Liu J, Fu X and Yang A: Identification of key genes and pathways in tongue squamous cell carcinoma using bioinformatics analysis. Med Sci Monit 23: 5924-5932, 2017.

22. Lim SY, Yuzhalin AE, Gordon-Weeks AN and Muschel RJ: Targeting the CCL2-CCR2 signaling axis in cancer metastasis. Oncotarget 7: 28697-28710, 2016.

23. Fang ZQ, Zang WD, Chen R, Ye BW, Wang XW, Yi SH, Chen W, $\mathrm{He} F$ and Ye G: Gene expression profile and enrichment pathways in different stages of bladder cancer. Genet Mol Res 12: 1479-1489, 2013.

24. Okai I, Wang L, Gong L, Arko-Boham B, Hao L, Zhou X, Qi X, $\mathrm{Hu} \mathrm{J}$ and Shao S: Overexpression of JAKMIP1 associates with Wnt/ $\beta$-catenin pathway activation and promotes cancer cell proliferation in vitro. Biomed Pharmacother 67: 228-234, 2013.

25. Hu X, Zhao Y, Wei L, Zhu B, Song D, Wang J, Yu L and Wu J: CCDC178 promotes hepatocellular carcinoma metastasis through modulation of anoikis. Oncogene 36: 4047-4059, 2017.

26. Alajez NM, Lenarduzzi M, Ito E, Hui AB, Shi W, Bruce J, Yue S, Huang SH, Xu W, Waldron J, et al: MiR-218 suppresses nasopharyngeal cancer progression through downregulation of survivin and the SLIT2-ROBO1 pathway. Cancer Res 71: 2381-2391, 2011

27. Ying J,Li H, Seng TJ, Langford C, Srivastava G, Tsao SW, Putti T, Murray P, Chan AT and Tao Q: Functional epigenetics identifies a protocadherin $\mathrm{PCDH} 10$ as a candidate tumor suppressor for nasopharyngeal, esophageal and multiple other carcinomas with frequent methylation. Oncogene 25: 1070-1080, 2006.

28. Cao JY, Liu L, Chen SP, Zhang X, Mi YJ, Liu ZG, Li MZ, Zhang H, Qian CN, Shao JY, et al: Prognostic significance and therapeutic implications of centromere protein $\mathrm{F}$ expression in human nasopharyngeal carcinoma. Mol Cancer 9: 237, 2010.

This work is licensed under a Creative Commons Attribution-NonCommercial-NoDerivatives 4.0 International (CC BY-NC-ND 4.0) License. 